\title{
REDES NEURAIS ARTIFICIAIS NA CLASSIFICAÇÃO DE FRUTOS: CE- NÁRIO BIDIMENSIONAL ${ }^{1}$
}

\author{
JEAN PAULO SILVA RAMOS ${ }^{2}$
}

\begin{abstract}
RESUMO - Inúmeras são as atividades agrícolas que necessitam de interação humana nos processos decisórios, e entre elas encontra-se a classificação de frutos. $\mathrm{O}$ consumo de frutos "in natura" exige altíssimo nível de qualidade, demandando um processo classificatório mais acurado. A classificação de frutos depende do reconhecimento de padrões natural ou artificial, de acordo com algumas categorias pré-definidas. Uma vez que um padrão de um fruto está sendo classificado, esse deve ser comparado com algum outro padrão armazenado. A maior parte da classificação de frutos é baseada na classificação humana.Este trabalho apresenta a possibilidade de uso de redes neurais artificiais no desenvolvimento de modelos de classificação de frutos por meio de vetores de padrões. Este trabalho foi desenvolvido no De-
\end{abstract}

\begin{abstract}
partamento de Máquinas Agrícolas da Faculdade de Engenharia Agrícola da Universidade Estadual de Campinas, as redes neurais armazenaram os vetores de padrões de frutos peso, diâmetro. Esses componentes vetoriais associados entre si interagiram, determinando um vetor padrão de saída de acordo com os padrões de frutos armazenados. Para atingir esses objetivos, foi $\mathrm{u}-$ sada uma rede Perceptron de múltiplas camadas, com algoritmo de treinamento tipo retro-propagação para armazenar os vetores de padrões de frutos e para classificação desses padrões de entrada. A rede treinada conseguiu aprender a relação entre vetores de entrada e saída, demonstrando a potencialidade do uso de tais ferramentas na classificação artificial.
\end{abstract}

TERMOS PARA INDEXAÇÃO: Redes Neurais Artificias, classificação, Redes Múltiplas Camadas.

\section{FRUIT SORTING USING ARTIFICIAL NEURAL NETWORKS: BIDIMENSIONAL CASE}

\begin{abstract}
Agriculture is one of the economic activities that more require the presence human being in the decision taking. Innumerable are the processes that require some type of human being interference in the conclusion of the processes. Fruit Sorting depends on human or artificial pattern recognition according to some pre defined categories. Once a fruit pattern is under classification, this one must be compared to some other ones stored. After that comparison it can be classified. Most sorting fruits jobs are human basis classification. This paper shows that using neural networks is possible to develop capable models of storing fruit pattern vectors. Given any fruit pattern vector to the model it can classify to the closest fruit pattern vector stored. The number of patterns were incremented and presented to the neural networks,
\end{abstract}

INDEX TERMS: Artificial Neural Networks, back-propagation, classifiers, sorting.

1. Parte da tese de doutorado apresentada pelo autor à UNIVERSIDADE ESTADUAL DE CAMPINAS/UNICAMP, área de Concentração: Máquinas Agrícolas.

2. Engenheiro Agrícola, M.Sc. em Engenharia Agrícola, área de Irrigação e Drenagem. Consultor - Microsoft Corporation - Av. Nações Unidas 12901, 27 andar - 04578-000 - São Paulo. jpsr@microsoft.com 


\section{INTRODUÇÃO}

Redes neurais têm seus princípios fundamentais assentados nos trabalhos de modelagem biológica de processos neurofisiológicos, cognitivos e comportamentais, freqüentemente identificadas como uma subespecialidade da Inteligência Artificial; outras vezes, como uma classe de modelos matemáticos para problemas de classificação e reconhecimento de padrões, outras, ainda, como uma parte da teoria conexionista dos processos mentais e finalmente como uma categoria de modelos em ciência de cognição (Kovacs, 1996).

A classificação natural de frutos é um bom exemplo da habilidade humana de realizar inferências, mediante características simples, tais como: peso, tamanho (raio, diâmetro), firmeza ("firmness"), cor, cheiro. Essas características permitem que uma dona-de-casa, em suas compras no supermercado, decida pela compra de um fruto exposto na prateleira, indicando, dessa forma, que esse específico fruto satisfaz os padrões de classificação dessa compradora.

Para o processo de classificação artificial de frutos, faz-se necessário o desenvolvimento de modelos que permitam estabelecer o relacionamento entre a entrada de padrões de classificação (Entradas), análise/processamento dessa informação e convergência para uma saída de classificação definida (Saída). A rede neural deve aprender a reconhecer padrões de entrada e definir a saída segundo classes definidas, ou seja, dado um determinado padrão de entrada, escolher em que categoria esse se enquadra melhor.

Conduziu-se este trabalho com o objetivo geral de desenvolver um modelo artificial de classificação de frutos que considere simultaneamente vários atributos de propriedades físicas durante o processo de classificação do fruto. Pelo uso das redes neurais artificiais, é possível classificar frutos utilizando a entrada simultânea de vetores de padrões. Os vetores de padrões contêm os valores das propriedades físicas peso e diâmetro, podendo conter valores de qualquer outra propriedade física. As redes neurais artificiais devem aprender a relação entre os padrões de entrada, que representam os valores das propriedades físicas e as classes desejadas na saída.

Geralmente a classificação de frutos e a aplicação de redes neurais estão relacionadas à maturidade de frutos. Se uma rede neural pode imitar exatamente quão maduro os compradores gostam de comprar seus tomates, pode-se, então, associar esse aspecto com conjuntos de modelos matemáticos que predizem quanto tempo será necessário até que eles cheguem a esse ponto de maturidade; dessa forma, as quitandas poderão comprar tomates com a cor ideal de venda, segundo a visão de seus compradores (Thai \& Shewfelt, 1991).

Thai \& Shewfelt (1991) afirmam que a inspeção das frutas e verduras é um procedimento importantíssimo na comercialização, armazenamento e processamento dos alimentos. Dentro da inspeção, um dos critérios mais importantes relacionados à qualidade dos alimentos é a cor, indicando maturação ou defeitos. A decisão na qualidade dos produtos varia entre inspetores e varia temporalmente.

Características de qualidade na compra, e que podem ser percebidas pelos sensos de tato, cheiro e/ou aparência visual, sem ingerir o produto, são muito importantes na diferenciação dos produtos na compra e preparação dos alimentos. Firmeza e cor são as características primárias na decisão de compra de pêssegos frescos e tomates (Thai \& Shewfelt, 1991).

Uma rede neural artificial (RNA) é um sistema de processamento de informação que possui algumas características de desempenho em comum com as redes neurais biológicas. Os modelos neurais artificiais têm como principal fonte de inspiração as redes neurais biológicas (Silva, 1998).

Bufo (2000) define uma rede neural como uma técnica matemática realizada, dentro de um fluxograma seqüencial de cálculo projetado, para obter resultados a partir de entradas de dados, independente da lei que rege esses resultados. As redes neurais artificiais oferecem recursos quando outros meios matemáticos podem ser impotentes.

Uma rede neural típica é constituída de uma camada de entrada de dados, uma camada de modificação desses dados chamada camada oculta ou escondida e uma camada de saída que são os resultados ou dados objetivos, como mostra a Figura 1. Cada camada é constituída de nódulos chamados de neurônios, nas quais ocorre a modificação dos dados segundo uma função de transferência com o campo de variação entre 0 e 1 . Essa função de transferência pode ser uma função sigmoidal ou senoidal, podendo até ser uma função linear, exemplificada na Figura 2. A camada de entrada recebe as variáveis, podendo ser um neurônio para cada variável, e cada variável assume diversos valores diferentes. Todos os neurônios de uma camada estão interligados com todos neurônios da camada subsequiente. Cada uma dessas interligações possui um peso $\mathbf{w}_{\mathrm{i}, \mathrm{j}}$, que multiplica a saída do neurônio anterior para gerar a entrada do neurônio subseqüente (Bufo, 2000). 
Rumelhart \& McClelland (1986), em pesquisas, demonstraram que é possível treinar redes neurais com camadas intermediárias, resultando no modelo de redes neurais artificiais mais utilizado atualmente: as redes Perceptron de múltiplas camadas (MLP), treinadas com o algoritmo retropropagação ("backpropagation").
Na Figura 3 verifica-se uma arquitetura do tipo MLP com duas camadas intermediárias. A rede apresentada como exemplo possui todas as conexões, o que significa que um neurônio em qualquer camada da rede está conectado a todas as outras unidades (neurônios) na camada anterior. O fluxo de sinais através da rede é feito positivamente, da esquerda para a direita, camada a camada.

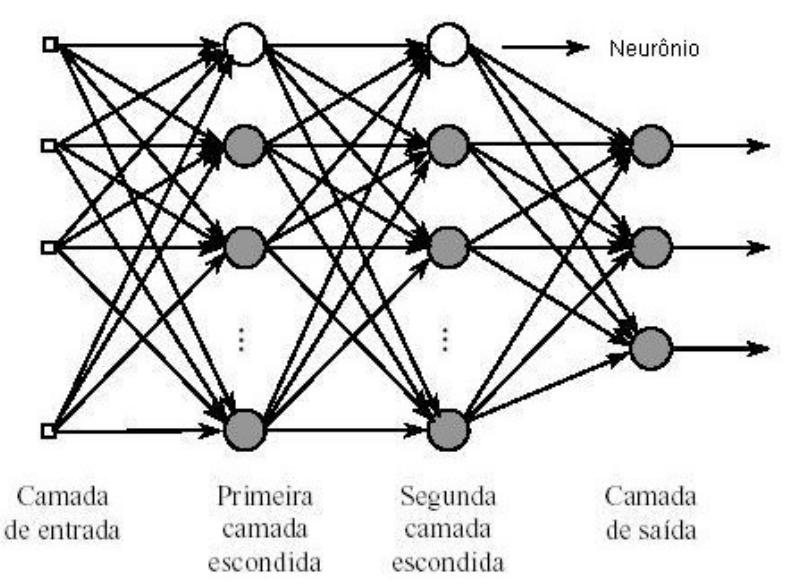

FIGURA 1 - Rede Neural de Múltiplas Camadas.

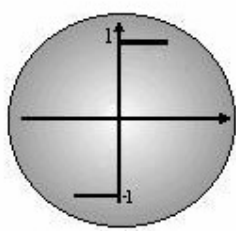

Função Degrau

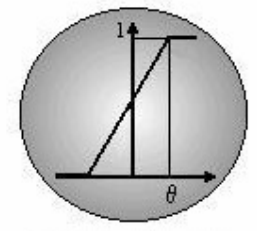

Threshold Logic

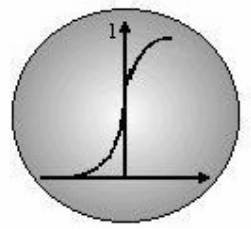

Sigmóide

FIGURA 2 - Funções de Transferência.

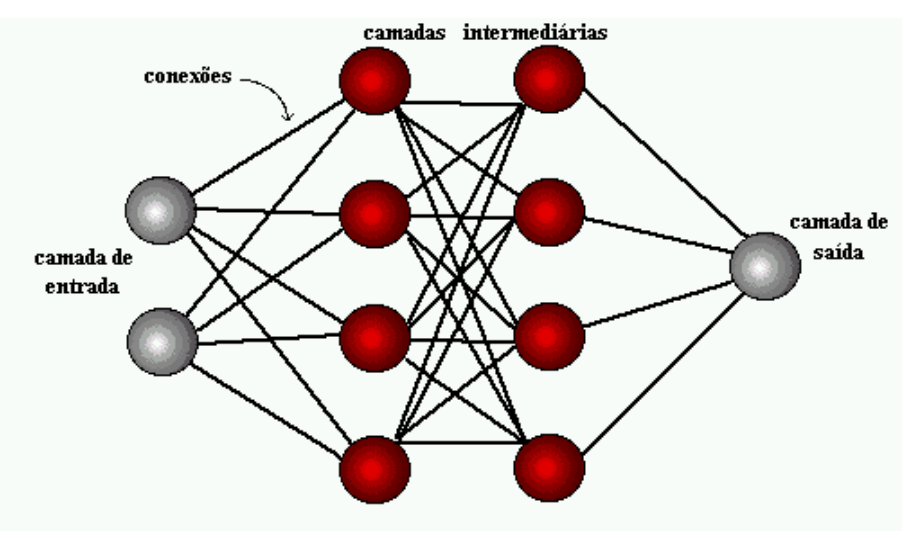

FIGURA 3 - Rede neural tipo MLP.

Ciênc. agrotec., Lavras. V.27, n.2, p.356-362, mar./abr., 2003 
Durante o treinamento com o algoritmo retropropagação ("backpropagation"), a rede opera em uma seqüência de alguns passos. Primeiro, um padrão é apresentado à camada de entrada da rede. A atividade resultante flui através da rede, camada por camada, até que a resposta seja produzida pela camada de saída. No segundo passo, a saída obtida é comparada à saída desejada para esse padrão particular. Se a saída obtida comparada com a saída desejada não estiver correta, o erro é, então, calculado. O erro é propagado a partir da camada de saída até a camada de entrada, e os pesos das conexões das unidades das camadas escondidas vão sendo modificados conforme o erro é retropropagado (Bufo, 2000).

\section{MATERIAL E MÉTODOS}

Este trabalho foi desenvolvido na Faculdade de Engenharia Agrícola da Universidade Estadual de Campinas, e todos os testes foram realizados em um computador PC Pentium III, $700 \mathrm{MHz}$, com 256 MB RAM, executando o Sistema Operacional Windows 2000 ® Professional. Todos os cenários testados neste trabalho tiveram convergência com intervalos de tempo inferior a duas horas e utilizou-se o programa de simulação SNNS. O SNNS (Stuttgart Neural Network Simulator) é um programa de simulação de redes neurais desenvolvido no Instituto para Sistemas Distribuídos e Paralelos da Universidade de Stuttgart. O simulador é gratuito para pesquisadores e pode ser obtido para "download" em: ftp.informatik.uni-stuttgart.de

O modelo de mapeamento de padrões proposto neste trabalho e exibido na Figura 4 permite que exista a combinação de duas propriedades simultaneamente e também computacionalmente paralelismo de informações. O mapeamento descrito na figura 4 é obtido pela medição de propriedades físicas de um conjunto amostral. Imagine um fruto que possui variação de peso de 180 g a 255 g: o uso de 4 bits para a representação binária indica $2^{4}$ ou 16 intervalos para a faixa de peso. Todos os frutos com peso de cada faixa de valores são mapeados para a classe binária de saída apropriada. A criação do espaço amostral da Figura 4 é determinada utilizando a medição das propriedades físicas e procedimento apresentado na tabela 1 , porém, para duas propriedades físicas simultaneamente: Peso e Diâmetro.

\begin{tabular}{|c|c|c|c|c|}
\hline Contínuo & Discreto & Resolução & Peso [g] & Classes \\
\hline 0 & 0000 & $180+(0 \times 5)$ & 180 & $A$ \\
\hline 1 & 0001 & $180+(1 \times 5)$ & 185 & Classe 1 \\
\hline 2 & 0010 & $180+(2 \times 5)$ & 190 & Binário: 00 \\
\hline 3 & 0011 & $180+(3 \times 5)$ & 195 & \\
\hline 4 & 0100 & $180+(4 \times 5)$ & 200 & \\
\hline 5 & 0101 & $180+(5 \times 5)$ & 205 & B \\
\hline 6 & 0110 & $180+(6 \times 5)$ & 210 & Classe 2 \\
\hline 7 & 0111 & $180+(7 \times 5)$ & 215 & Binário: 10 \\
\hline 8 & 1000 & $180+(8 \times 5)$ & 220 & \\
\hline 9 & 1001 & $180+(9 \times 5)$ & 225 & \\
\hline 10 & 1010 & $180+(10 \times 5)$ & 230 & C \\
\hline 11 & 1011 & $180+(11 \times 5)$ & 235 & Classe 3 \\
\hline 12 & 1100 & $180+(12 \times 5)$ & 240 & Binário: 01 \\
\hline 13 & 1101 & $180+(13 \times 5)$ & 245 & D \\
\hline 14 & 1110 & $180+(14 \times 5)$ & 250 & Classe 4 \\
\hline 15 & 1111 & $180+(15 \times 5)$ & 255 & Binário: 11 \\
\hline
\end{tabular}

TABELA 1 - Mapeamento de propriedades físicas e classes de saída. 
A análise simultânea das propriedades Peso e Diâmetro determina as áreas de classificação, que nada mais são do que o agrupamento dos vetores de mesma saída. Como exemplo no mapeamento (Figura 4) existem 4 classes, representadas no formato binário: Verde: [00], Azul-Escuro: [01], Vermelha: [10], Azul-Claro: [11].

A composição dos dois vetores lineares cria uma matriz bidimensional com definição de áreas de classificação. $\mathrm{O}$ vetor de entrada da rede será uma representação de um vetor de 8 Bits formado por 2 vetores individuais de 4 Bits, cada vetor representando uma proprie- dade física diferente. Os primeiros 4 bits correspondem à propriedade física Peso - [0011] e os últimos 4 bits correspondem à propriedade física Diâmetro - [0110]. Dessa forma, o vetor bidimensional resultante é o vetor [0011 0110]. O vetor resultante está mapeado para a classe [10].

A matriz bidimensional com o espaço de padrões proposto na figura 4 apresenta 4 classes diferentes com áreas de interesse não-regulares. Como pode ser observado existe, uma área de borda chamada de classe de descarte (classe verde [00]) simulando o mundo real.

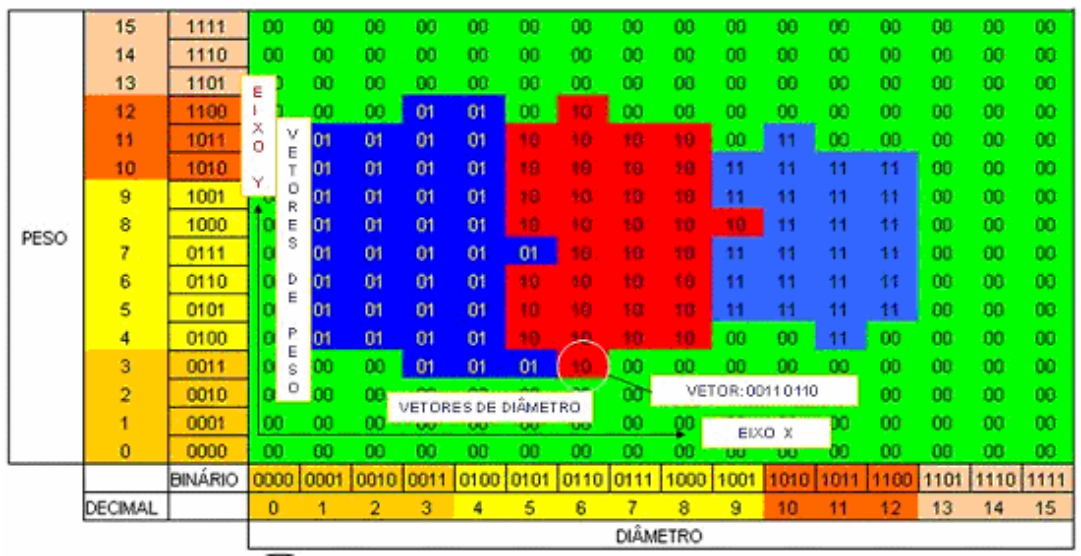

FIGURA 4 - Espaço Amostral/ Padrões bidimensional.

\section{Vetor resultante de 2 propriedades Físicas}

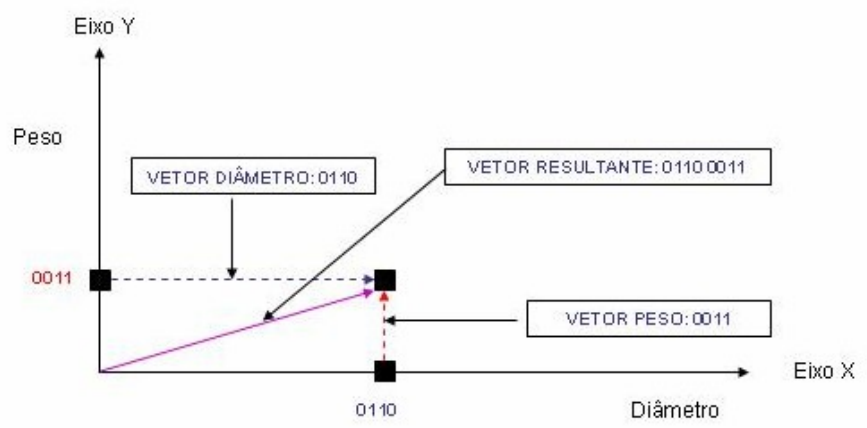

FIGURA 5 - Vetor Resultante de 2 propriedades físicas. 


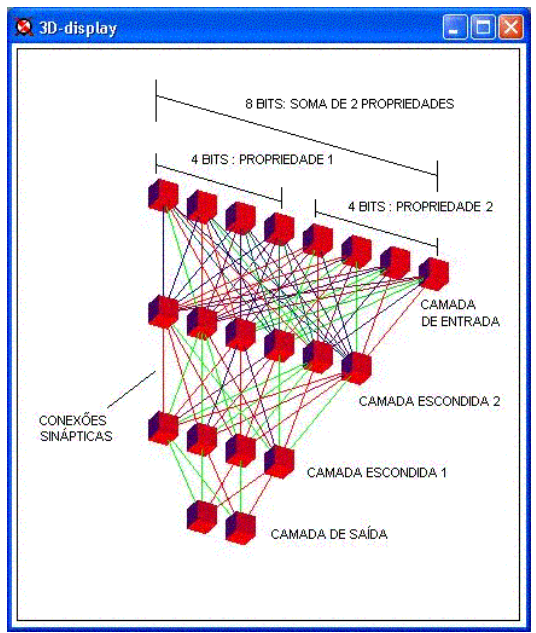

FIGURA 6 - Representação gráfica da topologia de uma rede do cenário bidimensional.

Baseando-se no mapeamento vetorial da Figura 5, foi criada uma rede neural que considera simultaneamente as propriedades físicas Peso e Diâmetro. Após inúmeros testes de convergência, a topologia final adequada à resolução do problema de classificação foi uma rede com 8 neurônios de entrada, 2 camadas escondidas com 2 neurônios cada uma e uma camada de saída com 2 neurônios. Na Figura 6 observa-se a topologia da rede bidimensional pesquisada para a classificação de frutos baseada em 2 propriedades físicas.

As redes foram treinadas e testadas com valores pertencentes ao espaço amostral descrito na Figura 4. A eficiência da rede foi calculada pela precisão da mesma, ou seja, por meio da divisão do número total de saídas corretas pelo número total de saídas analisadas:

$$
\mathrm{P}=(\mathrm{NVT}-\mathrm{NVD}) \mathrm{x} 100 / \mathrm{NVT}
$$

Em que:

$\mathrm{P}=$ precisão;

NVT = número de vetores totais testados;

$\mathrm{NVD}=$ número de vetores com diferença superior a 0.01 .

Nos testes feitos para o cenário bidimensional, o programa de simulação foi ajustado para treinamento das redes utilizando as funções de aprendizado tipo Retropropagação com termo de momento, atualização tipo
Ordem Topológica e inicialização tipo Pesos Randômi$\cos$.

Todos os vetores de entrada são compostos de 2 vetores lineares de 4 bits cada um, cujos componentes são valores booleanos pertencentes ao intervalo [0,1], representando a entrada de duas propriedades físicas, Peso e Diâmetro.

Os treinamentos foram realizados com todos os 256 vetores de entrada $\left(2^{8}\right)$ e respectivas classes desejadas de saída (Espaço vetorial - Figura 4), apresentados à rede seqüencialmente com a variação de um bit seqüencial por amostra. Os pesos foram inicializados em valores aleatórios.

Foram feitos 15 ciclos de treinamento usando variações incrementais de $10.000,50.000$ e 100.000 iterações. Foram utilizados vetores de 8 Bits para as entradas bidimensionais, e os neurônios 1 a 4 representam a entrada referente à propriedade física Peso, e os neurônios 5 a 8 representam a entrada referente à propriedade física Diâmetro. Foram utilizadas 4 camadas de neurônios.

\section{RESULTADOS E DISCUSSÃO}

A rede neural foi testada para o espaço amostral da figura 4. O gráfico da Figura 7 apresenta a dispersão de erros dos resultados da rede, em que se pode observar que apenas duas amostras tiveram resultados com diferença entre o resultado obtido e desejado superior 0,01 .

$\mathrm{O}$ valor inferior a 0,01 foi adotado como o limite para a diferença entre o valor obtido e o valor desejado. A precisão calculada para o espaço amostral estudado foi :

$$
\mathrm{P}=(256-2) \times 100 / 256->99.22 \%
$$

Analisando-se a eficiência da rede, observase que a rede consegue de forma efetiva fazer a classificação dos frutos com uma produtividade superior ao processo de classificação humana. $\mathrm{O}$ aspecto mais importante do uso de redes neurais na classificação dos frutos é a garantia do critério utilizado no processo, ou seja, o processo baseado na inferência humana introduz uma variação entre classificadores e varia temporalmente para o mesmo classificador. As redes neurais classificam baseadas no critério matemático, proporcionando sempre os resultados adequados para os quais foram treinadas, tornando-se um processo mais preciso. 


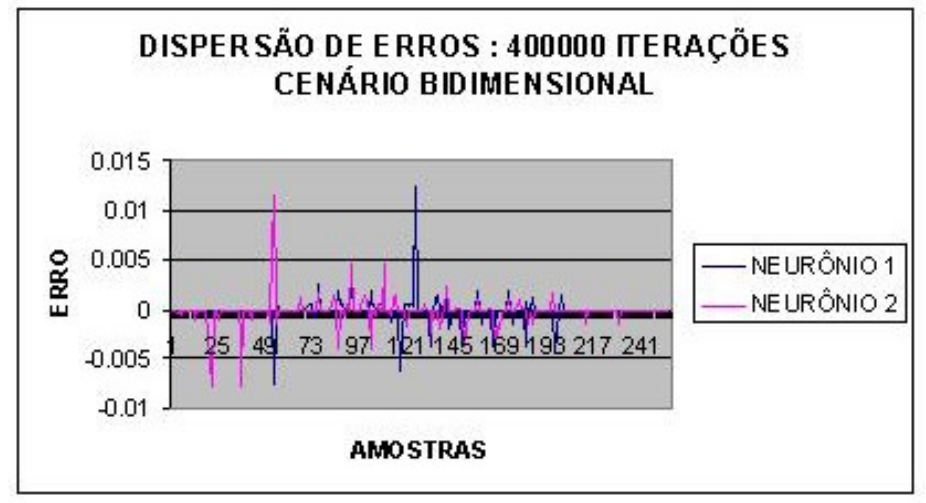

FIGURA 7 - Gráfico de Dispersão de Erros : 400.000 iterações.

\section{CONCLUSÕES}

Após o treinamento de 400.000 iterações, considerou-se que a rede neural de classificação bidimensional convergiu para um estado mínimo de energia, ou seja, a rede conseguiu estabelecer a correta correlação entre os dados de entrada e as classes de saídas desejadas. Dessa forma, com o modelo proposto de espaço vetorial e uso de redes neurais do tipo Perceptron de Múltiplas Camadas, considera-se que é possível a utilização desse tipo de ferramenta no auxílio à classificação artificial de frutos, melhorando significativamente a capacidade de classificação e diminuindo os erros causados pela variabilidade temporal e classificadores humanos.

\section{REFERÊNCIAS BIBLIOGRÁFICAS}

BUFO, M. J. Aplicação de rede neural artificial como auxiliar na predição do desempenho de um land farming. 2000. 192 p. Dissertação (Mestrado em En- genharia Agrícola) - Universidade Estadual de Campinas, Campinas.

KOVACS, Z. L. Redes neurais artificiais fundamentos e aplicações. São Paulo: Acadêmica, 1996. 174 p.

RUMElhart D. E.; McClELland J. L. Parallel distributed processing: Exploration in the Microstructure of Cognition. Cambridge: MIT, 1986. v. 1.

SILVA, L. N. C. Análise e síntese de estratégias de aprendizado para redes neurais artificiais. 1998. 210 p. Dissertação (Mestrado em Engenharia Elétrica) - Universidade Estadual de Campinas, Campinas.

THAI, C. N.; SHEWFELT, R. L. Modeling sensory color quality of tomato and peach: neural networks and statistical regression. Transactions of the ASAE, Saint Joseph, v. 34, n. 3, p. 950-954, 1991. 